\title{
Ki67 Proliferation Index in Germinal and Non- Germinal Subtypes of Diffuse Large B-Cell Lymphoma
}

Atif A. Hashmi ${ }^{1}$, Syeda N. Iftikhar ${ }^{1}$, Gul Nargus ${ }^{2}$, Omer Ahmed ${ }^{3}$, Ishaq Azeem Asghar ${ }^{4}$, Umme Aiman Shirazi ${ }^{1}$, Anoshia Afzal ${ }^{5}$, Muhammad Irfan ${ }^{6}$, Javaria Ali ${ }^{1}$

1. Pathology, Liaquat National Hospital and Medical College, Karachi, PAK 2. Pathology, Khyber Medical University, Peshawar, PAK 3. Internal Medicine, Liaquat National Hospital and Medical College, Karachi, PAK 4. Pathology, Ascension St. John Hospital, Detroit, USA 5. Pathology, University of Oklahoma Health Sciences Center, Oklahoma City, USA 6. Statistics, Liaquat National Hospital and Medical College, Karachi, PAK

Corresponding author: Atif A. Hashmi, atifhashmi345@gmail.com

\section{Abstract}

\section{Introduction}

Diffuse large B-cell lymphoma (DLBCL) is an aggressive B-cell lymphoma. The 2016 World Health Organization (WHO) update on hematopoietic tumors suggested that all DLBCL cases should be subtyped into germinal and non-germinal center phenotypes. Ki67 immunohistochemistry is a maker of cell proliferation and thus is used as a prognostic and predictive marker in various tumors of human body. Only a few studies evaluated the proliferative index of DLBCL subtypes in our population. Therefore, in this study, we evaluated the frequency of subtypes of DLBCL in our population and K67 index in each subtype.

\section{Methods}

A retrospective observational study was conducted in the Department of Histopathology, Liaquat National Hospital and Medical College, from January 2018 till December 2020, over a period of three years. A total of 101 cases with a histopathological diagnosis consistent DLBCL were included in the study. Immunohistochemical (IHC) stains CD10, B-cell lymphoma 6 (Bcl-6), and multiple myeloma oncogene 1 (MUM1) were applied for the further sub-categorization of DLBCL into germinal center B-cell-like (GCB) and non-GCB subtypes according to the Hans algorithm. The Ki67 index was interpreted in hot spots of the tumor and reported as an average percentage.

\section{Results}

Out of 101 DLBCL cases, $47.5 \%$ of DLBCL were GCB, while $52.5 \%$ were non-GCB subtypes. Bcl-2, Bcl-6, MUM1, c-Myc, CD10, and CD30 expression were noted in 62.4\%, 45.5\%, 42.6\%, 44.6\%, 39.6\%, and 7.9\% cases, respectively. The mean Ki67 index was $72.94 \pm 16.69 \%$. The mean Ki67 index in non-GCB-type DLBCL was $77.67 \pm 14.80 \%$, which was significantly higher than the mean Ki67 index in GCB-type DLBCL $(67.70 \pm 17.22 \%)$ with a significant $\mathrm{p}$-value $(\mathrm{p}=0.002)$. Cervical lymph node was the most common site of DLBCL, while the stomach was the most common extra-nodal site. A significant association of Ki67 index was noted with subtypes of DLBCL. A higher proportion of non-GCB-type DLBCL exhibited greater than $80 \%$ Ki67 index than GCB subtype DLBCL. Moreover, a significant association Ki67 index was noted with c-Myc positivity. A higher proportion of c-Myc-positive DLBCL had greater than 80\% Ki67 index.

(c) Copyright 2021

Hashmi et al. This is an open access article distributed under the terms of the Creative Commons Attribution License CC-BY 4.0., which permits unrestricted use, distribution, and reproduction in any medium, provided the original author and source are credited.

\section{Conclusion}

We found that non-GCB-type DLBCL had a higher Ki67 index than GCB subtype DLBCL, portending a poor prognostic significance of non-GCB subtype of DLBCL. Moreover, c-Myc expression was associated with a higher Ki67 index.

Categories: Pathology, Oncology, Hematology

Keywords: diffuse large b-cell lymphoma (dlbcl), germinal center b-cell-like (gcb), hans algorithm, ki67 index, proliferative index

\section{Introduction}

Diffuse large B-cell lymphoma (DLBCL) is the most common non-Hodgkin's lymphoma (NHL) worldwide. DLBCL is a high-grade BCL and can remain asymptomatic until a late disease stage, and symptoms largely depend upon the site of involvement. DLBCL has a propensity to involve any organ system, and can transform from low-grade BCL. The 2016 World Health Organization (WHO) update on hematopoietic tumors suggested that all DLBCL cases should be subtyped into germinal and non-germinal center phenotypes. The gene expression profiling studies divided DLBCL into germinal center B-cell-like (GCB) and activated B-cell-like (ABC) subtypes. The GCB subtype DLBCL has a better prognosis than ABC subtype 


\section{Cureus}

DLBCL [1]. Immunohistochemical (IHC) stains CD10, B-cell lymphoma 6 (Bcl-6), and multiple myeloma oncogene 1 (MUM1) serve as surrogate markers for gene profiling. The Hans IHC algorithm is a widely used approach to subtype DLBCL into GCB and non-GCB phenotypes. While the definite classification still rests on molecular-based gene profiling, WHO recognized that gene profiling is not universally accessible and therefore IHC-based categorization is a valid substitute. Ki67 immunohistochemistry is a marker of cell proliferation [2]. It has a wide utility in surgical pathology. Many studies have confirmed the diagnostic and prognostic role of Ki67 index in human cancers [3-5]. On the one hand, a high Ki67 index portends an aggressive nature of a tumor and hence poor prognosis; alternatively, the high proliferative index also leads to a better response to chemotherapy in many tumors. To our knowledge, only a few studies have evaluated the proliferative index of DLBCL subtypes in our population. Therefore, in this study, we evaluated the frequency of subtypes of DLBCL in our population and the Ki67 index in each subtype.

\section{Materials And Methods}

A retrospective observational study was conducted in the Department of Histopathology, Liaquat National Hospital and Medical College, from January 2018 till December 2020, over a period of three years. All cases were retrieved from the departmental archives. Total 101 cases with a histopathological diagnosis consistent DLBCL were included in the study. DLBCL was diagnosed on the basis of morphology and IHC profile. An IHC panel including CD20, PAX5, CD3, CD5, Tdt, cyclinD1 and CD23 was done to diagnose DLBCL.

Moreover, IHC stains CD10, Bcl-6, and MUM1 were applied for further sub-categorization of DLBCL. The Hans algorithm was applied for the subtyping. Cases with CD10 expression of more than $30 \%$, or more than $30 \%$ expression of Bcl- 6 without MUM1 expression (in the absence of CD10 expression) were classified as GCB subtype DLBCL. All other immunophenotypes were called non-GCB subtype DLBCL. The Ki67 index was interpreted in hot spots of the tumor and reported as an average percentage (Figures 1,2).

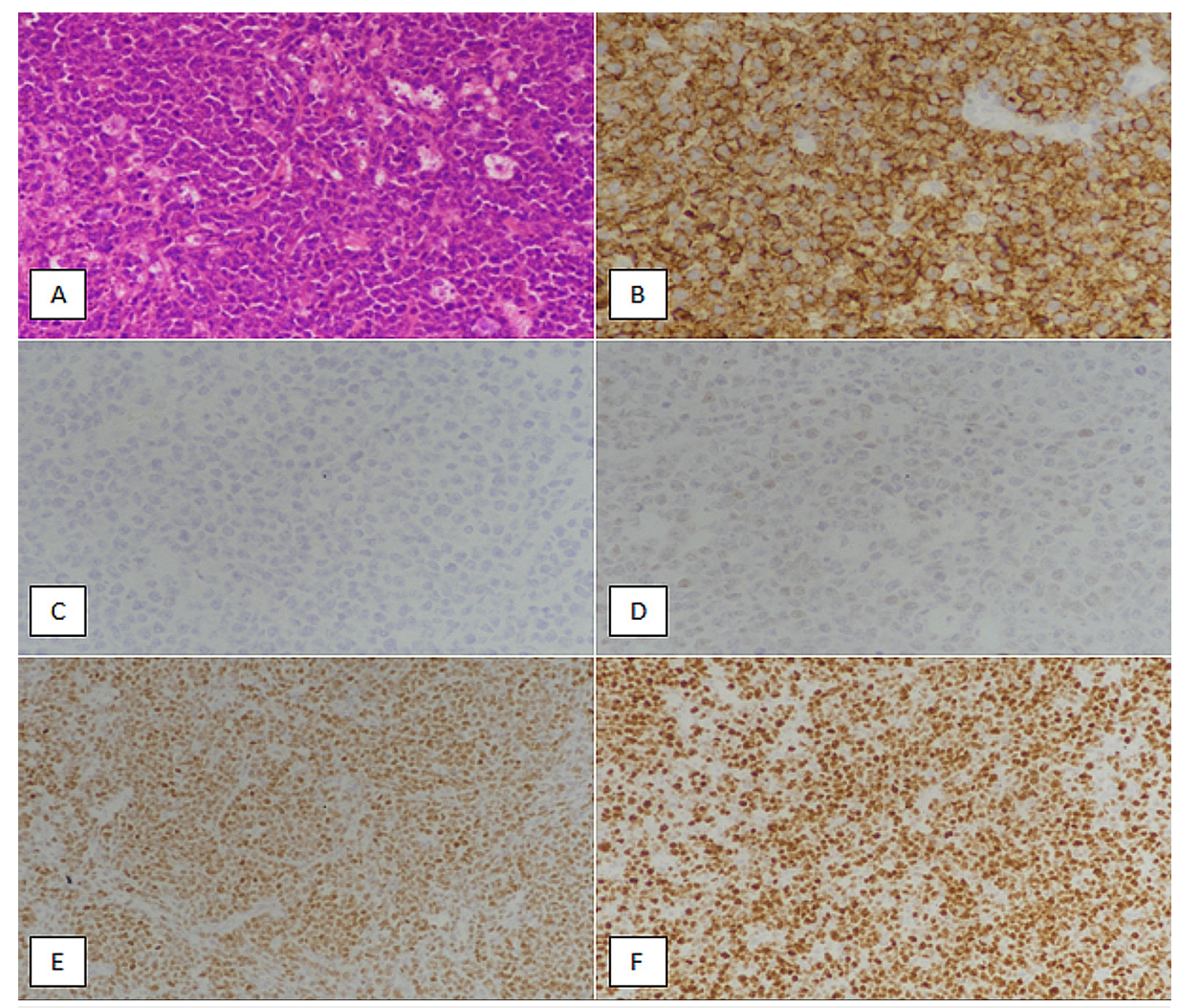

\section{FIGURE 1: Non-germinal center subtype (non-GCB) diffuse large B-cell lymphoma}

(A) Hematoxylin and eosin-stained section showing intermediate- to large-sized atypical lymphoid cells with scant cytoplasm arranged in sheets. (B) Tumor cells are diffusely positive (membranous expression) with CD20 IHC stain. (C) Tumor cells are negative with CD10 IHC marker. (D) Bcl-6 IHC staining showing less than $30 \%$ weak nuclear expression in tumor cells (more than $30 \%$ expression is needed to be interpreted as a positive expression). (E) More than $30 \%$ strong nuclear expression is noted with MUM1 IHC marker. (F) Ki67 IHC stain depicting more than $90 \%$ proliferative index in tumor cells.

IHC, immunohistochemical; Bcl-6, B-cell lymphoma 6; MUM1, multiple myeloma oncogene 1 


\section{Cureus}

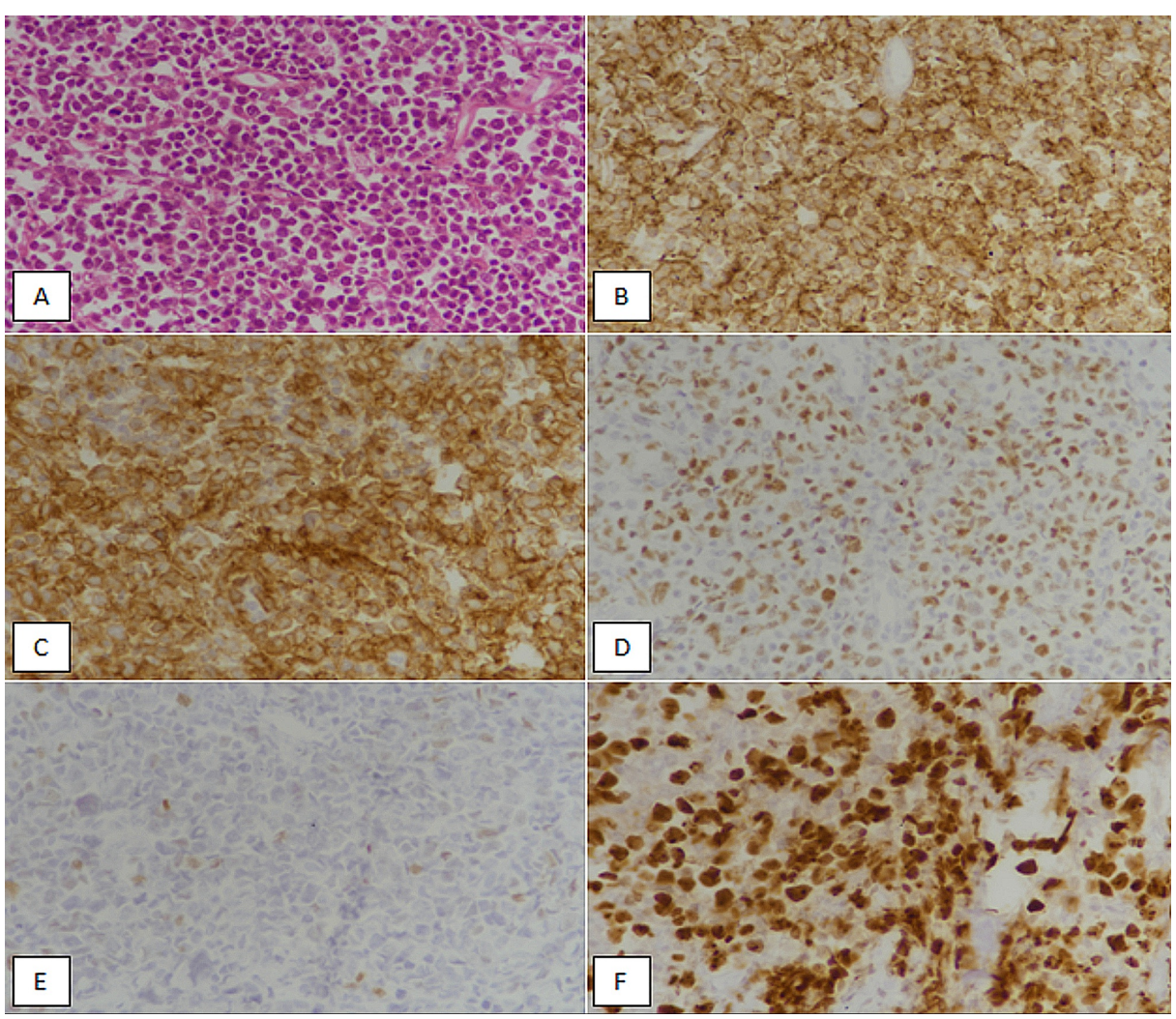

\section{FIGURE 2: Germinal center subtype (GCB) diffuse large B-cell lymphoma}

(A) Hematoxylin and eosin-stained section showing large-sized atypical lymphoid cells with scant cytoplasm in a sheet-like architecture. (B) Tumor cells are diffusely positive (membranous expression) with CD20 IHC stain. (C) Tumor cells are diffusely positive (membranous and cytoplasmic expression) with CD10 IHC marker. (D) Bcl-6 IHC staining showing nuclear expression in more than $30 \%$ tumor cells. (E) Less than $30 \%$ weak nuclear expression is noted with MUM1 IHC marker (more than $30 \%$ expression is needed to be interpreted as a positive expression). (F) Ki67 IHC stain depicting $75 \%$ proliferative index in tumor cells.

IHC, immunohistochemical; Bcl-6, B-cell lymphoma 6; MUM1, multiple myeloma oncogene 1

Data analysis was performed using Statistical Package for the Social Sciences, version 26.0 (IBM Corp., Armonk, NY). One-way analysis of variance (ANOVA) and Fisher's exact test were used to check the association. $p$-values $<0.05$ were considered as significant.

\section{Results}

The mean age of the patients was $52.91 \pm 16.71$ years and majority of the patients were more than 50 years of age. A total of $47.5 \%$ of DLBCL were GCB type, while $52.5 \%$ were non-GCB type; $59.4 \%$ of specimens were nodal. Bcl-2, Bcl-6, MUM1, c-Myc, CD10, and CD30 expression were noted in $62.4 \%, 45.5 \%, 42.6 \%, 44.6 \%$, $39.6 \%$, and $7.9 \%$ cases, respectively. The mean Ki67 index was $72.94 \pm 16.69 \%$ (Table 1 ).

\section{Clinicopathological characteristics}

Age (years)*

Age groups

$\leq 35$ years

36-50 years

$>50$ years

Gender

\section{Frequency (\%)}

$52.91 \pm 16.71$

$18(17.8)$

$20(19.8)$

63 (62.4)

$54(53.5)$ 


\section{Cureus}

Female

47 (46.5)

Subtype

Germinal center subtype

48 (47.5)

Non-germinal center subtype

$53(52.5)$

Site

Nodal

60 (59.4)

Extra-nodal

41 (40.6)

Specimen type

Trucut biopsy

46 (45.5)

Excision biopsy

55 (54.5)

Bcl-2

Positive

63 (62.4)

Negative

38 (37.6)

Bcl-6

Positive

46 (45.5)

Negative

55 (54.5)

MUM1

Positive

43 (42.6)

Negative

58 (57.4)

c-Myc

Positive

45 (44.6)

Negative

56 (55.4)

CD10

Positive

40 (39.6)

Negative

$61(60.4)$

CD30

Positive

8 (7.9)

Negative

93 (92.1)

Ki67 (\%) *

$72.94 \pm 16.69$

Ki67 groups

$\leq 40 \%$

$6(5.9)$

$41 \%-80 \%$

$62(61.4)$

$>80 \%$

$33(32.7)$

\section{TABLE 1: Descriptive statistics of the population under study}

Bcl, B-cell lymphoma 6; MUM1, multiple myeloma oncogene 1

*Mean \pm standard deviation

The mean Ki67 index in non-GCB-type DLBCL was $77.67 \pm 14.80 \%$, which was significantly higher than the mean Ki67 index in GCB-type DLBCL $(67.70 \pm 17.22 \%)$ with a significant $p$-value $(p=0.002)$. 


\section{Cureus}

Cervical lymph node was the most common site of DLBCL, while the stomach was the most common extranodal site. No significant association of Ki67 index was noted with the site of DLBCL (Table 2).

\begin{tabular}{|c|c|c|c|c|c|}
\hline \multirow[t]{2}{*}{ Site of DLBCL } & \multicolumn{4}{|c|}{ Ki67 index frequency (\%) } & \multirow{2}{*}{ p-value } \\
\hline & $\leq 40 \%$ & $41 \%-80 \%$ & $>80 \%$ & Total & \\
\hline Retroperitoneal lymph node & $1(16.7)$ & $4(6.5)$ & $1(3)$ & $6(5.9)$ & \multirow{21}{*}{0.625} \\
\hline Parotid gland & $0(0)$ & $1(1.6)$ & $0(0)$ & $1(1)$ & \\
\hline Mesenteric lymph node & $1(16.7)$ & $4(6.5)$ & 1 (3) & $6(5.9)$ & \\
\hline Cervical lymph node & $0(0)$ & $20(32.3)$ & $12(36.4)$ & $32(31.7)$ & \\
\hline Tonsil & $0(0)$ & $2(3.2)$ & $0(0)$ & $2(2)$ & \\
\hline Brain & $2(33.3)$ & $5(8.1)$ & $2(6.1)$ & $9(8.9)$ & \\
\hline Pancreas & $0(0)$ & $1(1.6)$ & $0(0)$ & 1 (1) & \\
\hline Stomach & 1 (16.) & $5(8.1)$ & $4(12.1)$ & $10(9.9)$ & \\
\hline Femur & $0(0)$ & $2(3.2)$ & $0(0)$ & $2(2)$ & \\
\hline Kidney & $0(0)$ & $1(1.6)$ & 1 (3) & $2(2)$ & \\
\hline Axillary lymph node & 1 (16./) & $6(9.7)$ & $2(0.1)$ & $9(8.9)$ & \\
\hline Nasopharynx & $0(0)$ & $1(1.6)$ & $1(3)$ & $2(2)$ & \\
\hline Liver & $0(0)$ & $2(3.2)$ & $0(0)$ & $2(2)$ & \\
\hline Inguinal lymph node & $0(0)$ & $4(6.5)$ & $3(9.1)$ & $7(6.9)$ & \\
\hline Large Intestine & $0(0)$ & $2(3.2)$ & $0(0)$ & $2(2)$ & \\
\hline Testes & $0(0)$ & $0(0)$ & 1 (3) & $1(1)$ & \\
\hline Ovary & $0(0)$ & $1(1.6)$ & $0(0)$ & $1(1)$ & \\
\hline Mediastinal lymph node & $0(0)$ & $0(0)$ & $2(6.1)$ & $2(2)$ & \\
\hline Soft palate & $0(0)$ & $0(0)$ & $1(3)$ & $1(1)$ & \\
\hline Spleen & $0(0)$ & $0(0)$ & 1 (3) & 1 (1) & \\
\hline Thyroid & $0(0)$ & $1(1.6)$ & $1(3)$ & $2(2)$ & \\
\hline
\end{tabular}

\section{TABLE 2: Ki67 index distribution with respect to individual sites of DLBCL}

DLBCL, diffuse large B-cell lymphoma

Fisher's exact test was applied.

A significant association of the Ki67 index was noted with subtypes of DLBCL. A higher proportion of nonGCB-type DLBCL exhibited greater than 80\% Ki67 index than GCB subtype DLBCL (Table 3). 


\section{Cureus}

\begin{tabular}{|c|c|c|c|c|}
\hline \multirow{3}{*}{ Subtype of DLBCL } & \multicolumn{3}{|c|}{ Ki67 index } & \multirow{3}{*}{ p-value } \\
\hline & \multicolumn{3}{|c|}{ Frequency (\%) } & \\
\hline & $\leq 40 \%$ & $41 \%-80 \%$ & $>80 \%$ & \\
\hline Germinal center subtype & $5(83.3)$ & $34(54.8)$ & $9(27.3)$ & \multirow{2}{*}{$0.005^{\star}$} \\
\hline Non-germinal center subtype & $1(16.7)$ & $28(45.2)$ & $24(72.7)$ & \\
\hline
\end{tabular}

\section{TABLE 3: Association of Ki67 index with subtype of DLBCL}

DLBCL, diffuse large B-cell lymphoma

Fisher's exact test was applied.

*p-value significant as $<0.05$.

Table 4 shows the association of Ki67 index with age, gender, site of involvement, and IHC expression. A significant association Ki67 index was noted with c-Myc positivity. A higher proportion of c-Myc-positive DLBCL had greater than $80 \% \mathrm{Ki} 67$ index. On the other hand, no significant association was noted with age, gender, site, or any other IHC marker expression.

\begin{tabular}{|c|c|c|c|c|}
\hline \multirow{2}{*}{ Clinicopathological characteristics and Immunohistochemical expression } & \multicolumn{3}{|l|}{ Ki67 index } & \multirow{3}{*}{ p-value } \\
\hline & \multicolumn{3}{|c|}{ Frequency (\%) } & \\
\hline & $\leq 40 \%$ & $41 \%-80 \%$ & $>80 \%$ & \\
\hline Age (years) ${ }^{\star}$ & $54.00 \pm 14.65$ & $52.96 \pm 16.83$ & $52.60 \pm 16.13$ & 0.962 \\
\hline \multicolumn{5}{|l|}{ Age groups ${ }^{\star \star}$} \\
\hline$\leq 35$ years & $1(16.7)$ & $12(19.4)$ & 5 (15.2) & \multirow{3}{*}{0.896} \\
\hline $36-50$ years & 2 (33.3) & $12(19.4)$ & $6(18.2)$ & \\
\hline$>50$ years & $3(50)$ & $38(61.3)$ & $22(66.7)$ & \\
\hline \multicolumn{5}{|l|}{ Gender $^{\star \star}$} \\
\hline Male & $3(50)$ & $34(54.8)$ & $17(51.5)$ & \multirow{2}{*}{0.946} \\
\hline Female & $3(50)$ & $28(45.2)$ & 16 (48.5) & \\
\hline \multicolumn{5}{|l|}{ Site } \\
\hline Nodal & $3(50)$ & 38 (61.3) & 19 (57.6) & \multirow{2}{*}{0.843} \\
\hline Extra-nodal & $3(50)$ & 24 (38.7) & $14(42.4)$ & \\
\hline \multicolumn{5}{|l|}{$\mathrm{Bcl}-2^{\star \star}$} \\
\hline Positive & 5 (83.3) & 37 (59.7) & 21 (63.6) & \multirow{2}{*}{0.625} \\
\hline Negative & 1 (16.7) & $25(40.3)$ & $12(36.4)$ & \\
\hline \multicolumn{5}{|l|}{ Bcl-6*^ } \\
\hline Positive & 2 (33.3) & $29(46.8)$ & 15 (45.5) & \multirow{2}{*}{0.848} \\
\hline Negative & $4(66.7)$ & 33 (53.2) & 18 (54.5) & \\
\hline \multicolumn{5}{|l|}{ MUM1 ${ }^{\star \star}$} \\
\hline Positive & $3(50)$ & $23(37.1)$ & 17 (51.5) & \multirow{2}{*}{0.320} \\
\hline Negative & $3(50)$ & 39 (62.9) & 16 (48.5) & \\
\hline C-Myc ${ }^{\star \star}$ & & & & \\
\hline
\end{tabular}




\section{Cureus}

\begin{tabular}{|c|c|c|c|c|}
\hline Positive & 1 (16.7) & $23(37.1)$ & 21 (63.6) & \multirow{2}{*}{$0.018^{\star \star \star}$} \\
\hline Negative & $5(83.3)$ & $39(62.9)$ & $12(36.4)$ & \\
\hline \multicolumn{5}{|l|}{ CD10*夫 } \\
\hline Positive & $4(66.7)$ & 27 (43.5) & $9(27.3)$ & \multirow{2}{*}{0.138} \\
\hline Negative & $2(33.3)$ & $35(56.5)$ & $24(72.7)$ & \\
\hline \multicolumn{5}{|l|}{ CD30** } \\
\hline Positive & $0(0)$ & $6(9.7)$ & $2(6.1)$ & \multirow{2}{*}{0.825} \\
\hline Negative & $6(100)$ & 56 (90.3) & 31 (93.9) & \\
\hline
\end{tabular}

\section{TABLE 4: Association of Ki67 index with clinicopathological characteristics and Immunohistochemical expression}

MUM1, multiple myeloma oncogene 1; Bcl, B-cell lymphoma

${ }^{*}$ Mean \pm standard deviation; analysis of variance was applied.

**Fisher's exact test was applied.

${ }^{\star * *} p$-value significant as $<0.05$.

\section{Discussion}

In this study, we assessed the Ki67 index in subtypes of DLBCL. We noted that non-GCB DLBCL had a higher mean Ki-67 index than GCB-type DLBCL. Moreover, c-Myc expression was associated with a higher Ki67 index.

Among various subtypes of NHL, DLBCL is the most common subtype, accounting for approximately $40 \%$ of NHL cases, with some cases having an inferior prognosis than others [6,7]. Ki67 is still considered a useful and reliable marker associated with prognosis, but some studies had mixed results associating high Ki67 expression with the DLBCL outcome and some showed an inverse relationship between the Ki67 index and clinical outcomes [8-11].

Other prognostic factors play an important role in determining the true nature of the behavior of DLBCL, including c-Myc expression, treatment with rituximab, and DLBCL subtype $[8,9,11]$. Tang et al. compared Ki67 and Bcl-2 as a combined tool to more accurately determine DLBCL prognosis and found it to be superior than using alone [12]. Some studies found Bcl-2 protein and c-Myc co-expression to be an independent risk factor indicating worse outcomes in DLBCL [13,14], but it might be related to the fact that c-Myc-positive DLBCL usually has a high Ki67 index as noted in our study. More studies are therefore required to differentiate among these factors and to delineate the Ki67 relationship with DLBCL prognosis to verify the previous contradicting/conflicting literature $[9,12,14,15]$.

He et al. found that the prognosis varied among different types of DLBCL after the introduction of rituximab and other targeted therapies, which can be important for clinical decision-making and for individualized treatment [16]. Ki67, which is a nuclear non-histone protein and is strictly associated with cell proliferation, is being widely used to monitor numerous malignancies, including lymphoma and neuroendocrine tumors $[9,17,18]$. Some recent papers have confirmed that a high Ki67 proliferation index is associated with shorter overall survival $[19,20]$. Consistent with our results, a high Ki67 index in non-GCB type of DLBCL was reported in other studies [9].

We view our study with a few limitations. First, follow-up of the patients was not available to compare survival differences in different subtypes of DLBCL. Clinical data regarding the stage of disease involvement and therapy-related information were not available to evaluate the response to chemotherapy in different Ki67-related subgroups of DLBCL. Therefore, we recommend large-scale clinical studies to assess the followup in subgroups of DLBCL based on the Ki67 index. Moreover, molecular studies were not done to evaluate gene rearrangements.

\section{Conclusions}

Subtyping of DLBCL into GCB and non-GCB is considered an essential pathological reporting parameter by WHO. We found a roughly equal proportion of GCB and non-GCB DLBCL in our study. We noted that nonGCB DLBCL had a significantly higher Ki67 index than GCB subtype DLBCL. Moreover, c-Myc expression was 
also associated with a higher Ki67 index. Ki67 index is a marker of tumor-cell proliferation and thus possesses both prognostic and predictive significance. The high Ki67 index in non-GCB-type DLBCL signifies a poor prognostic feature of this subtype of DLBCL.

\section{Additional Information \\ Disclosures}

Human subjects: Consent was obtained or waived by all participants in this study. Animal subjects: All authors have confirmed that this study did not involve animal subjects or tissue. Conflicts of interest: In compliance with the ICMJE uniform disclosure form, all authors declare the following: Payment/services info: All authors have declared that no financial support was received from any organization for the submitted work. Financial relationships: All authors have declared that they have no financial relationships at present or within the previous three years with any organizations that might have an interest in the submitted work. Other relationships: All authors have declared that there are no other relationships or activities that could appear to have influenced the submitted work.

\section{References}

1. Li S, Young KH, Medeiros LJ: Diffuse large B-cell lymphoma. Pathology. 2018, 50:74-87. 10.1016/j.pathol.2017.09.006

2. Miller I, Min M, Yang C, Tian C, Gookin S, Carter D, Spencer SL: Ki67 is a graded rather than a binary marker of proliferation versus quiescence. Cell Rep. 2018, 24:1105-1112.E5. 10.1016/j.celrep.2018.06.110

3. Hashmi AA, Hashmi KA, Irfan M, et al.: Ki67 index in intrinsic breast cancer subtypes and its association with prognostic parameters. BMC Res Notes. 2019, 12:605. 10.1186/s13104-019-4653-X

4. Haroon S, Hashmi AA, Khurshid A, Kanpurwala MA, Mujtuba S, Malik B, Faridi N: Ki67 index in breast cancer: correlation with other prognostic markers and potential in pakistani patients. Asian Pac J Cancer Prev. 2013, 14:4353-4358. 10.7314/apjcp.2013.14.7.4353

5. Hashmi AA, Hussain ZF, Faridi N, Khurshid A: Distribution of Ki67 proliferative indices among WHO subtypes of non-Hodgkin's lymphoma: association with other clinical parameters. Asian Pac J Cancer Prev. 2014, 15:8759-8763. 10.7314/apjcp.2014.15.20.8759

6. Moskowitz C: Diffuse large B cell lymphoma: how can we cure more patients in 2012? . Best Pract Res Clin Haematol. 2012, 25:41-47. 10.1016/j.beha.2012.01.008

7. Pileri SA, Agostinelli C, Sabattini E, et al.: Lymphoma classification: the quiet after the storm. Semin Diagn Pathol. 2011, 28:113-123. 10.1053/j.semdp.2011.02.001

8. Harris NL, Jaffe ES, Diebold J, Flandrin G, Muller-Hermelink HK, Vardiman J: Lymphoma classification from controversy to consensus: the R.E.A.L. and WHO Classification of lymphoid neoplasms. Ann Oncol. 2000, 11:S3-S10. 10.1093/annonc/11.suppl_1.S3

9. Li ZM, Huang JJ, Xia Y, et al.: High Ki-67 expression in diffuse large B-cell lymphoma patients with nongerminal center subtype indicates limited survival benefit from R-CHOP therapy. Eur J Haematol. 2012, 88:510-517. 10.1111/j.1600-0609.2012.01778.x

10. Hasselblom S, Ridell B, Sigurdardottir M, Hansson U, Nilsson-Ehle H, Andersson PO: Low rather than high Ki-67 protein expression is an adverse prognostic factor in diffuse large B-cell lymphoma. Leuk Lymphoma. 2008, 49:1501-1509. 10.1080/10428190802140055

11. Jerkeman M, Anderson H, Dictor M, Kvaløy S, Akerman M, Cavallin-Ståhl E: Assessment of biological prognostic factors provides clinically relevant information in patients with diffuse large B-cell lymphoma-a Nordic Lymphoma Group study. Ann Hematol. 2004, 83:414-419. 10.1007/s00277-004-0855-x

12. Tang YL, Zhou Y, Cheng LL, Su YZ, Wang CB: BCL2/Ki-67 index predict survival in germinal center B-celllike diffuse large B-cell lymphoma. Oncol Lett. 2017, 14:3767-3773. 10.3892/ol.2017.6577

13. Johnson NA, Slack GW, Savage KJ, et al.: Concurrent expression of MYC and BCL2 in diffuse large B-cell lymphoma treated with rituximab plus cyclophosphamide, doxorubicin, vincristine, and prednisone. J Clin Oncol. 2012, 30:3452-3459. 10.1200/JCO.2011.41.0985

14. Green TM, Young KH, Visco C, et al.: Immunohistochemical double-hit score is a strong predictor of outcome in patients with diffuse large B-cell lymphoma treated with rituximab plus cyclophosphamide, doxorubicin, vincristine, and prednisone. J Clin Oncol. 2012, 30:3460-3467. 10.1200/JCO.2011.41.4342

15. Akyurek N, Uner A, Benekli M, Barista I: Prognostic significance of MYC, BCL2, and BCL6 rearrangements in patients with diffuse large B-cell lymphoma treated with cyclophosphamide, doxorubicin, vincristine, and prednisone plus rituximab. Cancer. 2012, 118:4173-4183. 10.1002/cncr.27396

16. He X, Chen Z, Fu T, et al.: Ki-67 is a valuable prognostic predictor of lymphoma but its utility varies in lymphoma subtypes: evidence from a systematic meta-analysis. BMC Cancer. 2014, 14:153. 10.1186/14712407-14-153

17. Hashmi AA, Ali J, Yaqeen S, et al.: Clinicopathological features of primary neuroendocrine tumors of gastrointestinal/pancreatobiliary tract with emphasis on high-grade (Grade 3) well-differentiated neuroendocrine tumors. Cureus. 2020, 13:e12640. 10.7759/cureus.12640

18. Hashmi AA, Ali J, Khan K, et al.: Clinicopathological spectrum of primary and metastatic neuroendocrine neoplasms. Cureus. 2020, 12:e11764. 10.7759/cureus.11764

19. Zaiem F, Jerbi R, Albanyan O, Puccio J, Kafri Z, Yang J, Gabali AM: High Ki67 proliferation index but not cell-of-origin subtypes is associated with shorter overall survival in diffuse large B-cell lymphoma. Avicenna J Med. 2020, 10:241-248. 10.4103/ajm.ajm_81_20

20. Liu Y, Barta SK: Diffuse large B-cell lymphoma: 2019 update on diagnosis, risk stratification, and treatment . Am J Hematol. 2019, 94:604-616. 10.1002/ajh.25460 